“@ 2018 IEEE. Personal use of this material is permitted. Permission from IEEE must be obtained for all other uses, in any current or future media, including reprinting/republishing this material for advertising or promotional purposes, creating new collective works, for resale or redistribution to servers or lists, or reuse of any copyrighted component of this work in other works." 


\title{
Combining Skeletal Tracking and Virtual Reality for Game-based Fall Prevention Training for the Elderly
}

\author{
William L. Raffe Faculty of Engineering and Information Technology \\ University of Technology Sydney \\ Sydney, Australia \\ Email: William.Raffe@uts.edu.au Jaime A. Garcia Faculty of Engineering and Information Technology \\ University of Technology Sydney \\ Sydney, Australia \\ Email: Jaime.Garcia@uts.edu.au
}

\begin{abstract}
This paper provides a preliminary appraisal
of combining commercial skeletal tracking and virtual reality technologies for the purposes of innovative gameplay interfaces in fall prevention exergames for the elderly. This work uses the previously published StepKinnection game, which used skeletal tracking with a flat screen monitor, as a primary point of comparison for the proposed combination of these interaction modalities. Here, a Microsoft Kinect is used to track the player's skeleton and represent it as an avatar in the virtual environment while the HTC Vive is used for head tracking and virtual reality visualization. Multiple avatar positioning modes are trialled and discussed via a small self-reflective study (with the authors as participants) to examine their ability to allow accurate stepping motions, maintain physical comfort, and encourage self-identification or empathy with the avatar. While this is just an initial study, it highlights promising opportunities for designing engaging step training games with this integrated interface but also highlights its limitations, especially in the context of an unsupervised exercise program of older people in independent living situations.
\end{abstract}

I. INTRODUCTION

Falling over is a part of life, from childhood to adulthood, whether in a sporting environment or simply tripping while walking of losing one's balance while standing. While for children and young adults a simple accidental fall from a standing position may result in little more than grazes and bruises, as we get older a fall can result in serious injury or even death [1]. In Australia, for example, falls are the leading causes of death due to physical injury for those over the age of 85 [2].

However, it has been shown that staying active and performing regular leg and balance exercises can reduce the frequency of falls by up to $40 \%$ [3]. These exercises can be as simple as taking small steps in various directions while standing in a neutral posture [4]. However, identifying effective measures to motivate older individuals to perform these simple exercises remains an open research agenda [3]. 
For this reason, the StepKinnection game was previously designed, developed, and tested [5], [6] as both an entertaining form of exercise for independent living older people, as well as a novel means of collecting clinical data on stepping performance in the elderly. In that game, a Microsoft Kinect was used to track the position of players feet while the player is standing, feet-together in a neutral posture, and facing a computer monitor. On the monitor, the player can see a graphical representation of each foot from a top-down perspective. Virtual objects, such as bugs and coins, appear around the players standing position and the player is required to step on each one, returning to a neutral standing position after each step. The goal of the game is to get as many points as possible in progressively more challenging levels with objects that appear more frequently and disappear after a shorter period of time. The StepKinnection game being played by a user study participant can be seen in Figure 1. The StepKinnection game was demonstrated to be a successful step training tool, both in terms of improved stepping performance [5] and in terms of usability and engagement [6]. Both of these were verified through data collected during a user study with ten participants who played the game regularly over the course of three months.

Instead of designing a new game, the current line of inquiry aims at iterating on the StepKinnection game by making it more engaging and accessible to a wider audience through the identification of other potential interaction modalities. In doing so, we are attempting to balance multiple dimensions, including cost, usability, comfort, safety, engagement, immersion, and the ability to capture stepping motions accurately. Some of these dimensions are conflicting and it is a challenge to identify an interaction modality that optimizes all of them. Thus, this paper starts by examining a way of making the StepKinnection game more engaging and immersive, potentially at the expense of accessibility.

To that end, virtual reality is investigated as a way of increasing player engagement with the existing StepKinnection game. Here, virtual reality is defined in-line with the definitions outlined in [7], in which the player is fully immersed in the virtual environment via a head-mounted display with motion tracking. Thus, the skeletal tracking capabilities of the Microsoft Kinect are combined with the virtual reality visualization of the HTC Vive headmounted display.

There has been growing interest in combining technologies such as the Microsoft Kinect and the HTC Vive [8]-[10] but, to the best our knowledge, all of these papers are only initial works that do not discuss the potential avatar representation modes as we do here. Additionally, these papers do not address the specific utilization of this combined interaction modality for the purposes of games for health. There are also few papers that claim to combine the Kinect with "virtual reality" but in fact the term has been misused to refer to a typical digital environment displayed on a computer monitor or television, making the solutions presented in those papers more similar to the original StepKinnection game rather than the setup discussed here.

Thus, the primary contributions of this paper surrounds the preliminary appraisal of the combination of these two technologies. More specifically, this paper:

- Demonstrates a setup environment for evaluating the use of the Microsoft Kinect with the HTC Vive (see Section II).

- Identifies six modes for controlling and visualizing a virtual avatar of the player and evaluates them through an introspective study completed by the authors (see Section III).

- Discusses the general benefits and limitation of this interaction modality for the purposes of step training games for the elderly. 


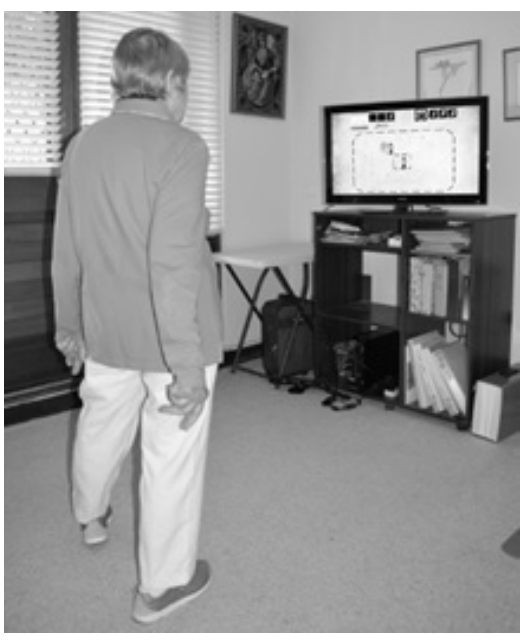

Fig. 1. The original StepKinnection game being played by a participant of the at-home three month user study.

The study performed here is small and utilizes the authors as participants but the insights generated are useful for examining the potential of combining skeletal tracking and virtual reality before investing in detailed game design and exposing the elderly target audience to an unknown gameplay interface. While this paper is aimed at games for the elderly and specifically for step training, the knowledge presented here is more generally useful for game design and exertion games [11] researchers.

\section{SETUP AND PROCESS}

A simple prototype was created to explore the potential combinations of skeletal tracking and a headmounted virtual reality display. This prototype consisted of a Microsoft Kinect ${ }^{1}$ for tracking the player's bodily movement, while the HTC Vive ${ }^{2}$ was used for the virtual reality display. Both of these devices use room scale tracking via infrared sensors. The Kinect use an infrared emitter and sensor built into a single device for

\footnotetext{
${ }^{1}$ Microsoft Kinect: https://developer.microsoft.com/en-us/windows/ kinect
}

${ }^{2}$ HTC Vive: https://developer.viveport.com/develop_portal/ detecting all objects within visible range and tracking relevant objects (e.g. human bodies) via computer vision. Meanwhile, the Vive has two "base-stations" that are placed at the corner of the rooms which emit the infrared light while the headset and hand controllers contain the sensors that are used to interpret their relative position to the base-stations. The play area for the following playtests was an empty space of $3 \mathrm{~m}$ by $3 \mathrm{~m}$, with an optimal distance of roughly $2.3 \mathrm{~m}$ from the Kinect sensor for a $1.82 \mathrm{~m}$ tall player.

The virtual world was created in the Unity Game Engine $^{3}$ and replicated the play area with a virtual floor similar in size to that of the physical space. The positional data provided by the Kinect and the Vive are accurate and similar enough that they could be mapped to the same virtual play area with little parameter tunning within the Unity editor. With the Vive head-mounted display being worn and the player facing the direction of the Kinect sensor, the player can see in front of them, on a wall at the edge of the virtual play area, the direct skeleton mapping generated by the Kinect, with the color image and depth sensor image of the Kinect display to the left and right. These were shown for debugging purposes and would likely not be used in a final prototype. Virtual replicas of Vive hand controllers were also shown, tracking the hand movement and input controls of the player as captured by the Vive. Similar to the raw Kinect data, the visualization of these controllers were only used for debugging purposes. The Kinect could also be used for tracking of hand motions and so the Vive hand controllers may not be needed in a final prototype.

The most important virtual representation for the below observations was a skeleton made up of spheres at key bodily joints as detected by the Kinect sensor,

\footnotetext{
${ }^{3}$ Unity Game Engine: https://unity3d.com/
} 


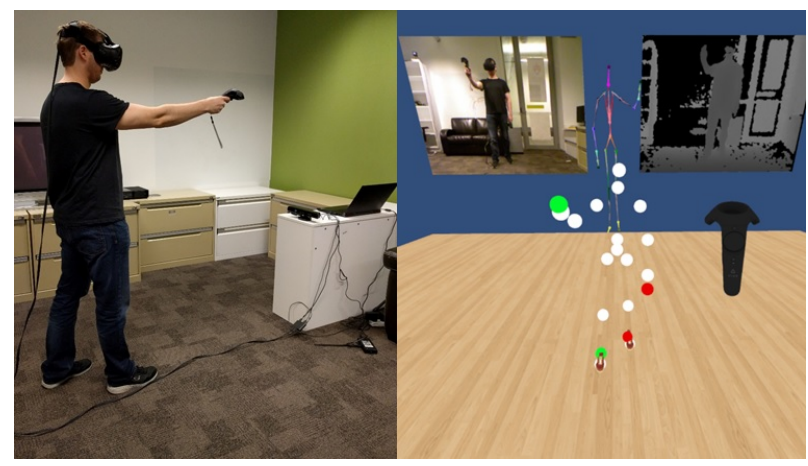

Fig. 2. The physical setup and the corresponding virtual scene as scene from the player's perspective. Green spheres represent the avatar's right hand and ankle while red spheres are the left hand and ankle.

which we refer to here as the player's "avatar". As the primary application being investigated here is for a stepping game, the avatar also has shoes to give a better sense of foot placement. In order to test stepping accuracy, primitive 3D shapes were added to the virtual environment around the avatar, allowing the player to control the avatar to step on the objects. This avatar, with potentially more visual fidelity to help the player identify or empathize with it, would likely be used in future work in which this interaction modality is used. Figure 2 shows the prototype in use, with both the physical and virtual images capturing the same moment in time.

With this setup in place, the key question being addressed here is how the avatar should be positioned relative to the player's view in virtual reality to give the player a positive experience. This is being addressed in the context of a step training game similar to the StepKinnection game, with the aim of maintaining or extending upon the usability and engagement qualities of that game [6] within this new interaction modality.

As this is an initial exploration of this interaction modality, it would be inappropriate to immediately engage elderly participants in an unknown experience. Instead, the following observations arose from introspective study performed by the authors in which each author took turns in isolation testing the six avatar visualization modes with think-aloud self-reporting, while an independent third party recorded key visual observations and conducted a post-play interview. Pair-wise comparison and discussion of the recorded observations was then done to find key shared insights. While it is acknowledged that this is not a comprehensive study, it proved suitable for discerning immediate benefits and limitations of both the avatar visualizations and the combination of the two technologies themselves.

\section{Avatar Visualization Modes}

The following six avatar visualization modes were tested to produce a variety of experiences. These modes are shown in Figure 3 and described in detail below. Other than the top-down mode, these were achieved by offsetting the avatar's starting position and rotation relative to the player's view in the virtual world.

Many of these modes have a similar analogue in traditional games (i.e. those that are not non-motion tracking or virtual reality based), such as first and third person views or top-down views. However, some modes (such as the mirror, mimic, and partner modes) are not as common in traditional games but are often seen in games designed for the Microsoft Kinect. However, as with most gameplay elements, we believe that the experience of these view modes is altered in the presence of a headmounted virtual reality display.

\section{A. Embodiment Mode}

This is a first-person perspective, with the positional tracking of the Kinect and Vive mapped as closely to each other in the virtual world as possible. This means that the player's view is positioned at the the top of the avatar's head, the Vive hand controllers can be seen at the end of the avatar's arms, and if the player looks down they can see their body and leg movements. 


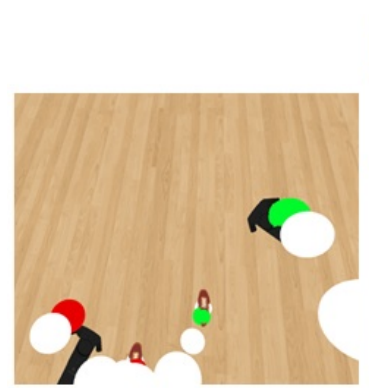

(a)
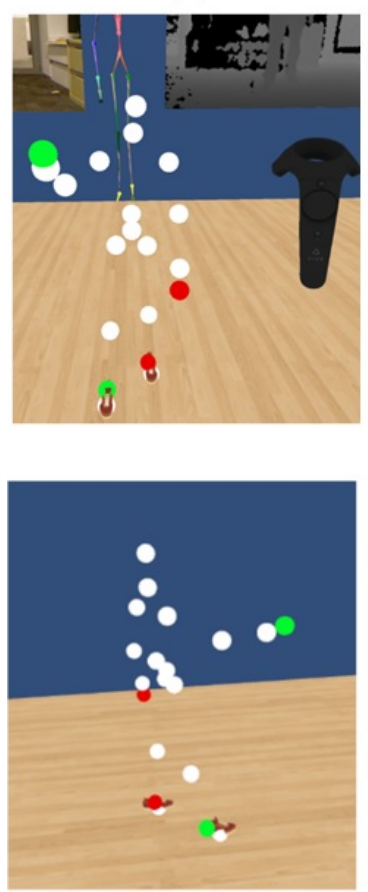

(e)

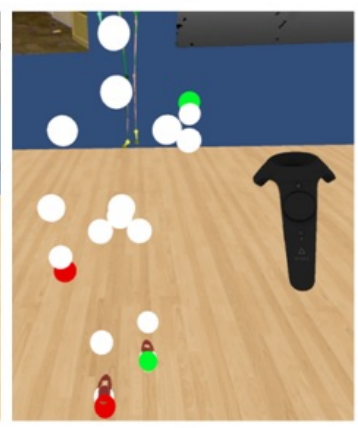

(d)

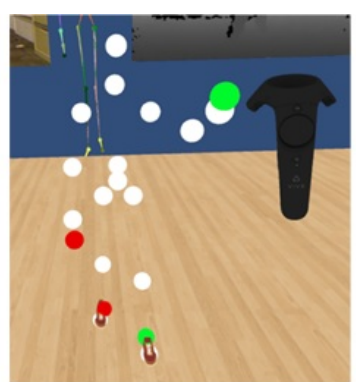

(b)

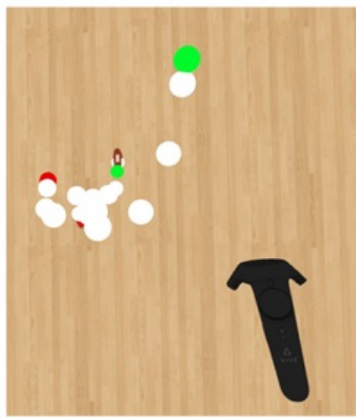

(f)
Fig. 3. The six primary avatar visualization modes that were trialled: (a) Embodiment, (b) Mirror, (c) Mimic, (d) Puppeteer, (e) Partner, and (f) Top-down. In all views, the player is standing as in Figure 2 with his right arm and right foot forward and head slightly turned to the right to see the Vive hand controller. In the Emobdiment mode, the player is looking down at their own feet with the right hand slightly raised. Green spheres represent the avatar's right hand and ankle while red spheres are the left hand and ankle.

The participants agreed that this was the most natural view in the virtual reality setting and gave a strong sense of presence and immersion. Perception errors in the body's proprioception, vestibular, and touch senses [12], combined with limits of depth perception in virtual reality [13], meant that the close mapping between the
Kinect and Vive data allowed the participants to feel that the avatar was a virtual embodiment of their physical self, even when the height of the avatar did not match that of the participant. This allowed the participants to make accurate movements in virtual world, which would be important for a step training game that required reasonably accurate stepping motions.

However, both participants identified neck strain after looking downwards for a while when they were simulating watching their feet stepping on virtual objects. This issue is exacerbated by the weight of the HTC Vive head-mounted display $(0.555 \mathrm{~kg})$. Both participants are healthy, physically active individuals in their 30's. Thus, this problem would likely be worse for older individuals for who the stepping program is designed. Additionally, it can be quite challenging to align the avatar with the player's actual hands and feet which requires lengthy tunning when compared to the other modes. While the errors in kinesthetic sense allowed for small alignment errors to go unnoticed, moderate alignment errors were more noticeable in this mode than in any other mode.

\section{B. Mirror Mode}

This mode is the equivalent of doing a stepping activity or exercise in-front of a mirror. The avatar is in-front of the player and facing the player at a distance at which the entire avatar skeleton can be seen. When the player, for example, raises their right hand, the avatar raises their left hand. This matches the default skeleton tracking of the Kinect that is shown in the debugging view directly in-front of the player.

This avatar control and visualization mode is a natural virtual analogue of performing physical exercises in front of a mirror. This may be good for some types of games where the avatar is simply used as an optional visual feedback mechanism where the player can evaluate their 
form by glancing at the avatar, as is often the case with exercising in the presence of a physical mirror.

However, for a game similar to StepKinnection where game elements to be stepped on would be placed around the avatar, this mode produces a learning curve due to the reverse of the forward and backward movement direction relative to the player. It also raises the question of whether it is an appropriate use of the technology; the skeletal tracking and virtual reality should enable activities, interactions, and visualizations that are not possible otherwise but in this case the sensation can more cheaply and accurately be achieved through the use of a physical mirror.

\section{Mimic Mode}

This mode is similar to the mirror mode above, with the avatar facing the player, except that the player's motions are not inverted across the central y-axis like a mirror. Instead, for example, when the player raises their right hand, the avatar also raises its own right hand. This is more similar to, for example, two people performing the same exercises while facing each other. This also matches the raw color and depth images that the Kinect camera is capturing and which are shown on the left and right debugging views at the far end of the virtual environment.

While technically this mode creates a more accurate binding between the player's and avatar's movements, the divergence from typical mirror optics produced somewhat of a cognitive dissonance for both participants. This meant that the participants had to maintain acute awareness of the control scheme in order to accurately step on virtual objects around the avatar. Thus, this mode was more difficult to control than the mirror mode.

Additionally, with the avatar staring at the player but not acting as a normal mirror image would, there was a lower sense of embodiment and it rather felt like facing another person who was perfectly matching movements and blocking the player's view forward. This felt confrontational to both participants, who said that it felt like the avatar could block their movement forward. In reality though, because of the inverted forward and backward directions as with the mirror mode, if the player did move too far forward from their starting position in physical space, then they would pass through the avatar until the avatar was behind them in virtual space.

\section{Puppeteer Mode}

In this mode, the avatar is again in front of the player but is facing the same direction as the player. Thus, none of the forward-backward or left-right axis are mirrored and so the player has direct control of the avatar. This is similar to the embodiment mode except that the player can see the entire avatar from directly behind it, controlling the avatar as if it were a puppet that is finely connected to the movements of the player.

The participants agreed that, other than the embodiment mode, this was the most natural control mode and allowed for the most accurate completion of simulated stepping tasks. Unlike the embodiment mode, however, this mode had the benefit of not causing neck strain as the player is able to keep a natural posture by constantly looking forward with only a slight tilt of the head to look at the feet of the avatar at a distance ahead of them. This mode also gave a strong sense of self-identification with the avatar, which could be enhanced by allowing the player to customize the avatar's appearance via physique adjustments or fashion choices.

\section{E. Partner Mode}

This mode similar to the puppeteer mode except that the avatar is located to the left or right of the player, giving the sense of the avatar being a partner who is 
performing the same motions. Both participants reported that rather than feeling that avatar was an embodiment of themselves, it instead generated feelings of companionship, as if there were another presence with them in the game, similar to the mimic mode above. However, both participants agreed that this was less confronting than the mimic mode due to being in a passive or cooperative position beside them rather than looking directly at them. This may be a desirable quality, especially as older individuals often report feelings of loneliness [14] .

However, similar to the embodiment mode, this caused neck strain due to constantly looking to one side of the body. Unlike the embodiment mode though, it was also reported by both participants to be hard to perform accurate stepping motions. Additionally, the player could rotate their entire body to face the avatar, which would cause them to enter the puppeteer mode but with the side of their body facing the Kinect sensor, which is more prone to errors in the skeletal tracking software.

As an adjustment, a half way point between the puppeteer and partner modes was tested, where the avatar appears at a $45^{\circ}$ angle to the player; slightly to the left and in front of the player and facing forward, giving an almost "over-the-shoulder" perspective common in commercial shooting and action games. Unfortunately, the predominant feeling with this adjustment was that there was an error in the setup and that the avatar had failed to be aligned properly with the player's position.

\section{F. Top-Down Mode}

The original StepKinnection game [5] displayed virtual representations of the player's feet on a monitor or television screen just behind the Kinect sensor. This representation was a top-down view, showing the top of virtual shoes and where they were moving on the horizontal plane of the play area. This allowed players to intuitively see where their physical feet were relative to virtual game elements arranged on this plane.

This same approach was trialled with the virtual reality setup, testing both a $90^{\circ}$ angle and a $45^{\circ}$ angle. For this, it was the player's view that was rotated and repositioned rather than the avatar to give a sense that the avatar was still grounded in the virtual world.

Both participants reported that this was the most disorienting avatar visualization mode. With the immersion of the HTC Vive virtual reality head set, both of the view angles gave the sense of an out-of-body experience, which was likely caused by the player's entire perspective of the world being rotated to an unnatural angle compared to typical human optical perception. The participants felt that the $90^{\circ}$ view gave less visual feedback of the motion of the avatar and a lower sense of self-identification with the avatar, resulting in a reduced sense of control. The $45^{\circ}$ view was received even worse, with the tilted view of the world resulting in the participants feeling like they were sliding backwards, especially when looking over their shoulder, and were observed as leaning forward to counter-balance this imaginary imbalance. Thus, this view of the avatar is more likely to induce falls then to train the player in their prevention.

\section{INTERACTION MODALITY BENEFITS AND}

\section{LIMITATIONS}

From the discussion of the various avatar visualization modes above, it is clear that there are some benefits of combining skeletal tracking with virtual reality for fall prevention training. Firstly, because of the virtual reality headset, there is a stronger sense of presence and immersion that can engage players in visually interesting environments. This is more so than the original StepKinnection game [6] that provided more of an abstract view of the player's actions and added engagement through accompanying thematic 2D graphics and audio. 
Secondly, while in the original StepKinnection players were easily able to recognize the virtual feet as being connected to their own, the combination of the Microsoft Kinect and the HTC Vive allows for players to see an entire virtual body that they can associate with. In modes such as the embodiment and puppeteer modes, this meant that there was an increased sense of self-identification with the avatar, which can potentially emotionally empower the player [15]. Meanwhile, in the partner mode it gave a sense of not being alone in the virtual world and enabled feeling of empathy rather than self-identification, which could be used to drive strong narratives or encourage further exercise through feelings of social connectedness [3].

Some of the visualization modes also gave a strong level of control over the avatar to accurately complete stepping activities. This was already a strong usability capability of the StepKinnection game [6], with its abstract top-down view of the player's feet. However, the various avatar modes described here, as well as potentially more that could be designed, highlight that the combination of these technologies allows for new perspectives on this type of game-based step training while also maintaining accurate motor control and task completion.

Unfortunately, there are also numerous disadvantages to the combination of these technologies, especially in the context of step training programs for older individuals. Firstly, the system setup itself is not accessible. The HTC Vive is an expensive piece of equipment that requires a high performance computer to function correctly. This potentially makes the entire setup prohibitively expensive for some users who are supporting themselves through a typical retirement fund or for distribution through a government funded initiative. Additionally, it has recently been announced that the Microsoft Kinect has been discontinued, so an alternative technology would need to be sourced. Even if the setup could be afforded, both technologies require an empty space to play safely and accurately that is likely larger than the empty space available in many homes. Finally, there is a reasonable level of technical competency required to set up and troubleshoot both devices.

In addition to the issues of accessibility are concerns regarding safety. The head-mounted virtual reality display is of an acceptable weight for typical commercial use. However, it may be too heavy for some older users, especially for prolonged or frequent exercise activities. When combined with the neck posture of modes such as the embodiment and partner modes, this weight could place undue stress on relatively weak muscles of the neck. Also, there is potential for users to become disoriented in the virtual space, especially if the floor of the virtual environment does not accurately match the position of the physical floor. In combination with the cable of the HTC Vive that can get wrapped around legs, this may actually increase the chances of a fall during step training for some users and make it more difficult to protect themselves from harm during the fall.

Finally, there was significant latency issues when both devices were in use. This is most noticeable in a delay with the Kinect's skeleton tracking and a ghosting effect created by the HTC Vive controllers when they are in motion. At first, it was thought that this may be due to the clashing infrared signals emitted and captured by both the Kinect and the Vive. However, when the Kinect emitters and sensors were blocked, the issue persisted. The issue could be associated with the computer's processing power. The setup was tested with both a moderately powerful laptop and a high performance gaming desktop; the skeletal tracking latency improved on the desktop but did not affect the Vive controller movement latency. The devices were then moved to USB3.0 ports on the desktop computer in an attempt to improve the communication 
rate of the peripheral devices. This improved the Vive controller latency slightly but ultimately the issue was not resolved entirely. This type of latency makes stepping tasks more difficult to complete efficiently, accurately, and safely.

\section{CONCLUSion AND Future Work}

Through the exercise of prototyping a simple setup that combines both skeletal tracking and virtual reality, evaluating it through introspection, and comparing the outcomes with the existing StepKinnection game, we have identified both advantages and disadvantages to this interaction modality for the purposes of a step training program for the elderly. The use of a virtual reality head-mounted display has the potential to increase the immersion of a gameplay experience and to increase feelings of self-identification or empathy with a virtual avatar. Some avatar visualization modes could potentially allow for more natural stepping challenges and allow for more efficient and accurate completion of these activities.

While we are confident that the issue of latency with the combined technologies could be resolved or incorporated into the game design, we currently do not foresee a means to overcome the issues relating to accessibility (such as financial, space availability, and technical knowledge requirements) and physical safety and well-being while using the setup. For this reason, we will continue to explore other potential game interaction modalities for fall prevention training. A simple adjustment to the current setup could be to remove the Kinect component and instead attach the Vive hand controllers (or similar) to the player's ankles. This reduces the cost and technical difficulty of the setup but still has issues around the accessibility and safety of virtual reality for the elderly as well as reduced position and rotation tracking of the player's feet.
Other alternatives include using dancing game mats that have shown to be effective in the past [16] or the use of augmented reality and computer vision techniques via cameras on mobile phones and tablets [17]. With dancing mats being inexpensive and mobile devices being widespread in the community, both of these alternatives increase the accessibility of a step training game. However, there may still be concerns regarding body posture if players are persistently looking at their feet or are required to aim a mobile device camera for extended periods of time. These approaches may also reduce immersion in the virtual environment or limit users to a discrete set of stepping motions. Ultimately, further innovation is required to design more engaging and accessible solutions for game-based fall prevention training programs.

\section{REFERENCES}

[1] G. Zijlstra, J. Van Haastregt, J. T. M. Van Eijk, E. van Rossum, P. A. Stalenhoef, and G. I. Kempen, "Prevalence and correlates of fear of falling, and associated avoidance of activity in the general population of community-living older people," Age and ageing, vol. 36, no. 3, pp. 304-309, 2007.

[2] A. B. of Statistics, "Underlying cause of death, selected causes by age at death, numbers and rates," Australian Demographic Statistics (3101.0), Dec 2016 Causes of Death (3303.0), Australia 2016, 2017.

[3] A. Barnett, B. Smith, S. R. Lord, M. Williams, and A. Baumand, "Community-based group exercise improves balance and reduces falls in at-risk older people: a randomised controlled trial," Age and ageing, vol. 32, no. 4, pp. 407-414, 2003.

[4] C. Sherrington, J. C. Whitney, S. R. Lord, R. D. Herbert, R. G. Cumming, and J. C. T. Close, "Effective exercise for the prevention of falls: A systematic review and meta-analysis," Journal of the American Geriatrics Society, vol. 56, no. 12, pp. 2234-2243, 2008.

[5] J. A. Garcia, D. Schoene, S. R. Lord, K. Delbaere, T. Valenzuela, and K. F. Navarro, "A bespoke kinect stepping exergame for improving physical and cognitive function in older people: A pilot study," Games for health journal, vol. 5, no. 6, pp. 382388, 2016.

[6] J. A. Garcia, W. L. Raffe, and K. Felix Navarro, "Assessing user engagement with a fall prevention game as an unsupervised 
exercise program for older people," in Proceedings of the Australasian Computer Science Week (ACSW 2018). ACM, 2018, to appear, pp. 1-8.

[7] P. Milgram and F. Kishino, "A taxonomy of mixed reality visual displays," IEICE TRANSACTIONS on Information and Systems, vol. 77, no. 12, pp. 1321-1329, 1994.

[8] F. Fririksson, H. Kristjánsson, D. Sigursson, D. Thue, and H. Vilhjálmsson, "Become your avatar: fast skeletal reconstruction from sparse data for fully-tracked vr,' in Proceedings of the 26th international conference on artificial reality and telexistence and the 21st eurographics symposium on virtual environments: posters and demos. Eurographics Assoc. Arkansas, Little Rock, 2016.

[9] M.-W. Hsu, T.-Y. Wu, Y.-C. Wu, Y.-A. Chen, Y.-C. Lin, and P.-S. $\mathrm{Ku}$, "Party animals: Creating immersive gaming experience for physically co-present vr and non-vr players," in Proceedings of the 2017 CHI Conference Extended Abstracts on Human Factors in Computing Systems. ACM, 2017, pp. 222-225.

[10] M. Tanaya, K. Yang, T. Christensen, S. Li, M. O’Keefe, J. Fridley, and K. Sung, "A framework for analyzing ar/vr collaborations: An initial result," in Computational Intelligence and Virtual Environments for Measurement Systems and Applications (CIVEMSA), 2017 IEEE International Conference on. IEEE, 2017, pp. 111-116.

[11] F. Mueller, D. Edge, F. Vetere, M. R. Gibbs, S. Agamanolis, B. Bongers, and J. G. Sheridan, "Designing sports: a framework for exertion games," in Proceedings of the SIGCHI Conference on Human Factors in Computing Systems. ACM, 2011, pp. 2651-2660.

[12] I. Rock and J. Victor, "Vision and touch: An experimentally created conflict between the two senses," Science, vol. 143, no. 3606, pp. 594-596, 1964.

[13] N. Padmanaban, R. Konrad, T. Stramer, E. A. Cooper, and G. Wetzstein, "Optimizing virtual reality for all users through gaze-contingent and adaptive focus displays," Proceedings of the National Academy of Sciences, 2017.

[14] L. Liu, Z. Gou, and J. Zuo, "Social support mediates loneliness and depression in elderly people," Journal of health psychology, vol. 21, no. 5, pp. 750-758, 2016.

[15] R. Pugliese, K. Förger, and T. Takala, "Game experience when controlling a weak avatar in full-body enaction," in International Conference on Intelligent Virtual Agents. Springer, 2015, pp. 418-431.

[16] G. Pichierri, K. Murer, and E. D. de Bruin, "A cognitive-motor intervention using a dance video game to enhance foot placement accuracy and gait under dual task conditions in older adults: a randomized controlled trial," BMC geriatrics, vol. 12, no. 1, p. 74, 2012.

[17] J. A. Garcia and K. F. Navarro, "The mobile rehapp: an ar- based mobile game for ankle sprain rehabilitation," in Serious Games and Applications for Health (SeGAH), 2014 IEEE 3rd International Conference on. IEEE, 2014, pp. 1-6. 